\title{
BOVINE RESPIRATORY DISEASE IN FINLAND
}

\author{
Nikunen S. ${ }^{1}$, Härtel H., Neuvonen E., Tanskanen R., Kivelä S-L., Salmela P., Pyörälä S. \\ and Saloniemi $H$.
}

${ }^{1}$ Karjaportti, PL 60, FI-50101 Mikkeli, Finland. E-mail: sanna.nikunen@karjaportti.fi

\begin{abstract}
Aim of the study
Aim of this study was to obtain basic knowledge of pathogens that cause bovine respiratory tract disease complex in Finland.

\section{Materials and methods}

The study was conducted in 1998-1999. It included 18 cattle herds which had problems with bovine respiratory tract disease complex. Four to five diseased calves from each farm were chosen for closer examination. A thorough clinical examination and tracheobronchial lavage were done. Blood samples were taken from the calves at the acute phase and 3-4 weeks later. In addition, 10 blood samples from cattle of different age were taken from the same herds.

Because bovine herpesvirus-1 (BHV-1) does not exist in Finland and also bovine virus diarrhoea virus (BVDV) is very rare, serum samples were tested for antibodies to bovine parainfluenza virus3 (PIV-3), bovine respiratory syncytial virus (BRSV), bovine coronavirus (BCV), bovine adenovirus-3 (BAV-3) and bovine adenovirus-7 (BAV-7). Some samples were also tested for antibodies to BVDV. ELISA-test was used for antibodies against PIV-3, BRSV, BCV and BVDV. Virus neutralization test was used for BAV-3 and BAV-7. Bacteria were cultured from lavage fluid and in vitro susceptibility for selected antimicrobials (ampicillin, penicillin, trimethoprim-sulfamethoxazole, gentamicin, tetracycline and ciprofloxacin) tested. One calf was autopsied.
\end{abstract}

\section{Results}

Antibodies to PIV-3 were found in all 18 herds (100\%). Antibodies to BCV were discovered from $16 / 18$ herds (89\%). Antibodies to BRSV were found from 12/18 herds (67\%) but these positive findings were all individual animals. Antibodies to BAV-3 and BAV-7 were observed from 15/18 farms $(83 \%)$ and $18 / 18$ farms (100\%) respectively. Antibodies to BVDV were not detected at all (0/6).

Bacterial growth was found in 19 samples $(22 \%)$ at 10 herds $(56 \%)$. The most common finding was Pasteurella sp., which was monitored from 14 samples (74\% of positive samples) at 8 herds (44\%). All 14 Pasteurella $s p$. strains were in vitro susceptible to all antimicrobials tested.

All 18 herds had mycoplasma findings; Mycoplasma dispar was found from 17 herds (94\%) and Mycoplasma sp. from 16 herds (89\%). All colonies that were examined using immunofluorescence test proved to be M. bovirhinis colonies.

\section{Conclusions}

PIV-3 and BCV appeared to be common pathogens in Finland, and also BAV-3 and BAV-7 were significant pathogens. BRSV was a infrequent finding. Tracheobronchial lavage revealed only few pathogens and Pasteurella (now Mannheimia) haemolytica, Haemophilus somnus and Mycoplasma bovis were not found. (H. somnus was cultured from the lungs of the autopsied calf.) The most common bacterial findings were Pasteurella sp. and M. dispar. 